\title{
Divergent and Stereocontrolled Synthesis of the Enamide Side Chains of Oximidines I/II, Salicylihal- amides A/B, Lobatamides A/D, and CJ-12,950
}

\author{
Robert S. Coleman* and Pei-Hua Liu \\ Department of Chemistry, The Ohio State University, 100 West $18^{\text {th }}$ Avenue, Columbus, Ohio 43210
}

\section{Supporting Information}

General Methods: Proton and carbon NMR spectra were recorded on Bruker DRX-400 and DRX-500 spectrometers. Chemical shifts are reported in ppm relative to the chloroform peak at $7.24 \mathrm{ppm}\left({ }^{1} \mathrm{H}\right)$ or $77.0 \mathrm{ppm}\left({ }^{13} \mathrm{C}\right)$, or the acetone peak at $2.04 \mathrm{ppm}\left({ }^{1} \mathrm{H}\right)$ or 29.8 and $206.0 \mathrm{ppm}\left({ }^{13} \mathrm{C}\right)$. Assignments in the ${ }^{1} \mathrm{H}$ NMR spectra were made using ${ }^{1} \mathrm{H}$ COSY and ${ }^{1} \mathrm{H} /{ }^{1} \mathrm{H}$ decoupling experiments. Mass spectroscopy was performed by the Ohio State University Chemistry Mass Spectrometry Facility on a Micromass QTOF2 instrument using electrospray (ESI) or electron impact (EI) ionization. Infrared (IR) spectra were recorded on a Perkin-Elmer $1600 \mathrm{FT}$-IR spectrometer as neat samples on $\mathrm{NaCl}$ plates. Unless otherwise specified, all reactions were run under an inert atmosphere of nitrogen. Solvents were freshly distilled before use. Yields refer to isolated material determined to be pure by NMR spectroscopy and thin layer chromatography (TLC), unless specified otherwise in the text.

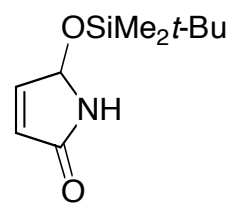

5-(tert-Butyldimethylsilyloxy)-1,5-dihydro-2-pyrrolone (7). 5-Hydroxy-3-pyrrolin-2one $(770 \mathrm{mg}, 7.80 \mathrm{mmol})$ was dissolved in DMF $(10 \mathrm{~mL})$ at room temperature. Imidazole $(1.60 \mathrm{~g}, 23.3$ $\mathrm{mmol})$ and tert-butyldimethylsilyl chloride $(2.60 \mathrm{~g}, 17.2 \mathrm{mmol})$ were added and the reaction mixture was stirred at $25^{\circ} \mathrm{C}$ for $12 \mathrm{~h}$. The reaction was quenched by the addition of cold $\left(0{ }^{\circ} \mathrm{C}\right)$ saturated $\mathrm{NaCl}$ and the mixture was extracted with ethyl acetate $(3 \times 30 \mathrm{~mL})$. The combined organic extracts were washed with saturated aqueous $\mathrm{NH}_{4} \mathrm{Cl}(3 \times 60 \mathrm{~mL})$, dried $\left(\mathrm{Na}_{2} \mathrm{SO}_{4}\right)$, and concentrated. The residue was purified by flash chromatography $(5 \times 8 \mathrm{~cm}$ silica, $1: 1 \mathrm{EtOAc} /$ hexane $)$ to afford pure $7(1.4 \mathrm{~g}, 85 \%)$ as a white solid: ${ }^{1} \mathrm{H} \mathrm{NMR}\left(\mathrm{CDCl}_{3}, 400 \mathrm{MHz}\right) \square 7.18(\mathrm{br}, 1 \mathrm{H}, \mathrm{NH}), 6.86-6.84(\mathrm{~m}, 1 \mathrm{H}, \mathrm{C} 4-\mathrm{H})$ [in the presence of $\mathrm{D}_{2} \mathrm{O}$ (acetone- $\left.\mathrm{d}_{6}\right)$, changes to: $\left.7.01(\mathrm{dd}, 1 \mathrm{H}, J=5.7,1.5 \mathrm{~Hz}, \mathrm{C} 4-\mathrm{H})\right], 6.06(\mathrm{dd}, 1 \mathrm{H}, J=5.7,0.6$ $\mathrm{Hz}, \mathrm{C} 3-\mathrm{H}), 5.68$ (br d, $1 \mathrm{H}, J=0.5 \mathrm{~Hz}, \mathrm{C} 5-\mathrm{H}), 0.86\left(\mathrm{~s}, 9 \mathrm{H},\left(\mathrm{CH}_{3}\right)_{3} \mathrm{C}\right), 0.12\left(\mathrm{~s}, 3 \mathrm{H}, \mathrm{CH}_{3} \mathrm{Si}\right), 0.10(\mathrm{~s}, 3 \mathrm{H}$, $\left.\mathrm{CH}_{3} \mathrm{Si}\right) ;{ }^{13} \mathrm{C} \mathrm{NMR}\left(\mathrm{CDCl}_{3}, 100 \mathrm{MHz}\right) \square 172.8,148.6,127.7,81.1,25.6$ 18.0, -4.2, -4.4; IR (neat) $\square_{\max }$ $3277,2930,2859,1711,1436,1349,1256,1233,1106,881,832,782,686 \mathrm{~cm}^{-1}$; HRMS (ESI), $m / z$ 236.1082 (calcd for $\mathrm{C}_{10} \mathrm{H}_{19} \mathrm{NO}_{2} \mathrm{Si}+\mathrm{Na}$ : 236.1077). 
<smiles>CC(C)C/C=C\I</smiles>

(Z)-1-Iodo-4-methyl-1-pentene [(Z)-2]. Iodomethyltriphenylphosphonium iodide (40 g, 85 $\mathrm{mmol})$ was slurried in THF $(80 \mathrm{~mL})$ at room temperature in a reaction flask shielded from light with aluminum foil, and a solution of $\mathrm{NaN}\left(\mathrm{SiMe}_{3}\right)_{2}(1.0 \mathrm{M}$ in THF, $76 \mathrm{~mL}, 85 \mathrm{mmol})$ was added dropwise by syringe pump over $30 \mathrm{~min}$. After stirring at this temperature for $10 \mathrm{~min}$, the reaction mixture was cooled to $-60{ }^{\circ} \mathrm{C}$, and HMPA $(32 \mathrm{~mL}, 204 \mathrm{mmol})$ was added in one portion, and the reaction mixture was cooled to $-78{ }^{\circ} \mathrm{C}$. Isovaleraldehyde $(6.5 \mathrm{~mL}, 68.4 \mathrm{mmol})$ was added neat by syringe over $5 \mathrm{~min}$. The cold bath was removed, and stirring was continued for $1 \mathrm{~h}$. The reaction mixture was diluted with hexane $(50 \mathrm{~mL})$ was washed with saturated $\mathrm{NaHCO}_{3}$, saturated $\mathrm{NaCl}$, and saturated $\mathrm{NH}_{4} \mathrm{Cl}$, and was dried $\left(\mathrm{Na}_{2} \mathrm{SO}_{4}\right)$ and concentrated. The residue was purified by flash chromatography $(5 \times 5 \mathrm{~cm}$ silica, hexane) to afford the desired vinyl iodide $(7.9 \mathrm{~g}, 56 \%)$ as a colorless oil as a 25:1 Z/E ratio of olefins, as determined by ${ }^{1} \mathrm{H}$ NMR. Vinyl iodide $(Z)-2$ was characterized: ${ }^{1} \mathrm{H}$ NMR $\left(\mathrm{CDCl}_{3}, 500 \mathrm{MHz}\right) \square 6.22$ $(\mathrm{dt}, 1 \mathrm{H}, J=7.3,1.0 \mathrm{~Hz}, \mathrm{C} 1-\mathrm{H}), 6.16(\mathrm{dt}, 1 \mathrm{H}, J=7.3,7.0 \mathrm{~Hz}, \mathrm{C} 2-\mathrm{H}), 2.03(\mathrm{ddd}, 2 \mathrm{H}, J=7.0,6.8,1.0$ $\mathrm{Hz}, \mathrm{C} 3-\mathrm{H}), 1.78-1.73$ (m, 1H, C4-H), 0.93 (d, 6H, $J=6.7 \mathrm{~Hz}, \mathrm{C} 5-\mathrm{H}, \mathrm{C} 6-\mathrm{H}) ;{ }^{13} \mathrm{C} \mathrm{NMR}\left(\mathrm{CDCl}_{3}, 125\right.$ $\mathrm{MHz}) \square$ 140.3, 82.9, 43.5, 27.8, 22.3; IR (neat) $\square_{\max } 2958,1609,1465,1385,1368,1305,1262,1168$, 1096, 825, 679, $623 \mathrm{~cm}^{-1}$; HRMS (EI), $m / z 209.9897$ (calcd for $\mathrm{C}_{6} \mathrm{H}_{11} \mathrm{I}: 209.9899$ ).

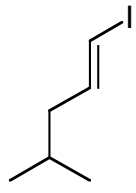

(E)-1-Iodo-4-methyl-1-pentene [(E)-2]. Anhydrous $\mathrm{CrCl}_{2}(5.00 \mathrm{~g}, 40.7 \mathrm{mmol})$ was slurried in THF $(50 \mathrm{~mL})$ at $0{ }^{\circ} \mathrm{C}$. A solution of isovaleraldehyde $(0.75 \mathrm{~mL}, 6.8 \mathrm{mmol})$ and $\mathrm{CHI}_{3}(5.40$ $\mathrm{g}, 13.6 \mathrm{mmol})$ in THF $(20 \mathrm{~mL})$ was added dropwise by cannula. After stirring at $0{ }^{\circ} \mathrm{C}$ for $3 \mathrm{~h}$, the reaction mixture was poured onto water $(200 \mathrm{~mL})$ and the mixture was extracted with hexane $(3 \times 20 \mathrm{~mL})$. The combined organic extracts were dried $\left(\mathrm{Na}_{2} \mathrm{SO}_{4}\right)$ and concentrated. The residue was purified by flash chromatography $(1.5 \times 10 \mathrm{~cm}$ silica, hexane $)$ to afford the desired vinyl iodides $(0.6 \mathrm{~g}, 41 \%)$ as colorless oil in a 4:1 E/Z ratio, as determined by ${ }^{1} \mathrm{H}$ NMR. Vinyl iodide $(E)-2$ was characterized: ${ }^{1} \mathrm{H}$ $\operatorname{NMR}\left(\mathrm{CDCl}_{3}, 400 \mathrm{MHz}\right) \square 6.48(\mathrm{dt}, 1 \mathrm{H}, J=14.3,7.5 \mathrm{~Hz}, \mathrm{C} 2-\mathrm{H}), 5.94(\mathrm{dt}, 1 \mathrm{H}, J=14.3,1.3 \mathrm{~Hz}, \mathrm{C} 1-\mathrm{H})$, 1.92 (ddd, 2H, $J=7.5,7.2,1.3 \mathrm{~Hz}, \mathrm{C} 3-\mathrm{H}), 1.68-1.62$ (m, 1H, C4-H), 0.87 (d, 6H, J = 6.7 Hz, C5-H, $\mathrm{C} 6-\mathrm{H}) ;{ }^{13} \mathrm{C} \mathrm{NMR}\left(\mathrm{CDCl}_{3}, 125 \mathrm{MHz}\right) \square 145.7,75.0,45.2,27.8,22.1$.

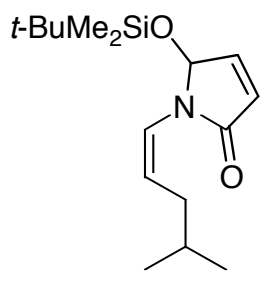

(Z)-5-(tert-Butyldimethylsilyloxy)-1-(4-methyl-1-pentenyl)-1,5-dihydro-2-pyrrolone $[(Z)-8]$. Vinyl iodide $(Z)-2(210 \mathrm{mg}, 1.00 \mathrm{mmol})$ was dissolved in dioxane $(10 \mathrm{~mL})$ at room temperature in a reaction flask shielded from light with aluminum foil. The reaction mixture was deoxygenated with a stream dry $\mathrm{N}_{2}$ for $15 \mathrm{~min}$. Copper (I) thiophenecarboxylate (CuTc) (19.1 mg, 0.1 $\mathrm{mmol})$, trans- $N, N \exists$ dimethyl-1,2-cyclohexanediamine $(0.35 \mathrm{~mL}, 0.20 \mathrm{mmol})$, pyrrolone 1 (320 mg, 1.50 $\mathrm{mmol}$ ), and $\mathrm{K}_{3} \mathrm{PO}_{4}\left(425 \mathrm{mg}, 2.0 \mathrm{mmol}\right.$ ) were added successively under $\mathrm{N}_{2}$. The reaction flask was sealed and the reaction mixture was warmed at $90{ }^{\circ} \mathrm{C}$ for $24 \mathrm{~h}$. The resulting red-brown slurry was cooled to room temperature, diluted with ether $(50 \mathrm{~mL})$, and was washed with $\mathrm{pH} 7.0$ $\mathrm{KH}_{2} \mathrm{PO}_{4} / \mathrm{Na}_{2} \mathrm{HPO}_{4}$ buffer $(3 \times 10 \mathrm{~mL}, 1.0 \mathrm{M})$. The organic layer was dried $\left(\mathrm{Na}_{2} \mathrm{SO}_{4}\right)$ and concentrated. The residue was purified by flash chromatography $(3 \times 10 \mathrm{~cm}$ silica, 1:5 EtOAc/hexanes $)$ to afford pure 
(Z)-8 (168.1 mg, 56\%) as a colorless oil: ${ }^{1} \mathrm{H} \mathrm{NMR}\left(\mathrm{CDCl}_{3}, 500 \mathrm{MHz}\right) \square 6.88(\mathrm{dd}, 1 \mathrm{H}, J=6.0,1.6 \mathrm{~Hz}$, $\mathrm{C} 4-\mathrm{H}), 6.13(\mathrm{~d}, 1 \mathrm{H}, J=6.0, \mathrm{~Hz}, \mathrm{C} 3-\mathrm{H}), 6.0(\mathrm{dt}, 1 \mathrm{H}, J=8.9,1.7, \mathrm{C} 1 \mathrm{H}), 5.71$ (br d, 1H, $J=0.5 \mathrm{~Hz}, \mathrm{C} 5-$ $\mathrm{H}), 5.23(\mathrm{dt}, 1 \mathrm{H}, J=8.9,7.2 \mathrm{~Hz}, \mathrm{C} 2 \exists \mathrm{H}), 2.05-2.02(\mathrm{~m}, 2 \mathrm{H}, \mathrm{C} 3 \mathrm{H}), 1.66-1.61(\mathrm{~m}, 1 \mathrm{H}, \mathrm{C} 4 \exists \mathrm{H}), 0.88(\mathrm{~d}$, $6 \mathrm{H}, \mathrm{J}=7.2 \mathrm{~Hz}, \mathrm{C} 5 \mathrm{H}, \mathrm{C} 6 \mathrm{H}), 0.86\left(\mathrm{~s}, 9 \mathrm{H},\left(\mathrm{CH}_{3}\right)_{3} \mathrm{C}\right), 0.07\left(\mathrm{~s}, 3 \mathrm{H}, \mathrm{CH}_{3} \mathrm{Si}\right), 0.05\left(\mathrm{~s}, 3 \mathrm{H}, \mathrm{CH}_{3} \mathrm{Si}\right) ;{ }^{13} \mathrm{C}$ $\operatorname{NMR}\left(\mathrm{CDCl}_{3}, 125 \mathrm{MHz}\right) \square 168.1,146.5,128.6,123.7,120.4,85.1,36.9,28.8,26.0,22.9,22.8,18.5$, $-3.3,-3.7$; IR (neat) $\square_{\max } 3441,2949,1718,1655,1414,1366,1256,1121,984,838,780,726 \mathrm{~cm}^{-1}$; HRMS (ESI), $m / z 318.1854$ (calcd for $\mathrm{C}_{16} \mathrm{H}_{29} \mathrm{NO}_{2} \mathrm{Si}+\mathrm{Na}$ : 230.1860).

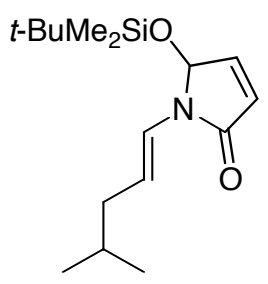

(E)-5-(tert-Butyldimethylsilyloxy)-1-(4-methyl-1-pentenyl)-1,5-dihydro-2-pyrro-

lone $[(\boldsymbol{E})-\mathbf{8}]$. Following the procedure for the preparation of $(Z)-8$, pyrrolone $7(234 \mathrm{mg}, 1.1 \mathrm{mmol})$ and vinyl iodide $(E)-\mathbf{2}(176 \mathrm{mg}, 0.84 \mathrm{mmol})$ afforded $(E)-8(178.6 \mathrm{mg}, 72 \%)$ as a colorless oil: ${ }^{1} \mathrm{H}$ NMR $\left(\mathrm{CDCl}_{3}, 400 \mathrm{MHz}\right) \square 6.88(\mathrm{dd}, 1 \mathrm{H}, J=6.0,1.4 \mathrm{~Hz}, \mathrm{C} 4-\mathrm{H}), 6.60(\mathrm{~d}, 1 \mathrm{H}, J=14.6 \mathrm{~Hz}, \mathrm{C} 1 \boxminus \mathrm{H}), 6.13$ (dd, $1 \mathrm{H}, J=6.0,0.6 \mathrm{~Hz}, \mathrm{C} 3-\mathrm{H}), 5.73$ (br dd, $1 \mathrm{H}, J=0.8,0.6 \mathrm{~Hz}, \mathrm{C} 5-\mathrm{H}), 5.33$ (dt, 1H, $J=14.6,7.4 \mathrm{~Hz}, \mathrm{C} 2 \mathrm{G}$ $\mathrm{H}), 1.94-1.90(\mathrm{~m}, 2 \mathrm{H}, \mathrm{C} 3 \mathrm{H}), 1.62-1.55(\mathrm{~m}, 1 \mathrm{H}, \mathrm{C} 4 \mathrm{H}), 0.87(\mathrm{~d}, 6 \mathrm{H}, J=6.7 \mathrm{~Hz}, \mathrm{C} 5 \boxminus \mathrm{H}, \mathrm{C} 6 \boxminus \mathrm{H}), 0.86$ $\left(\mathrm{s}, 9 \mathrm{H},\left(\mathrm{CH}_{3}\right)_{3} \mathrm{C}\right), 0.08\left(\mathrm{~s}, 3 \mathrm{H}, \mathrm{CH}_{3} \mathrm{Si}\right), 0.03\left(\mathrm{~s}, 3 \mathrm{H}, \mathrm{CH}_{3} \mathrm{Si}\right) ;{ }^{13} \mathrm{C} \mathrm{NMR}\left(\mathrm{CDCl}_{3}, 100 \mathrm{MHz}\right) \square 166.9$, 146.0, 127.8, 121.8, 112.0, 83.5, 39.9, 28.8, 25.5, 22.3, 22.1, 17.9, -3.5, -3.7; IR (neat) $\square_{\max } 3163$, 2943, 1718, 1668, 1464, 1398, 1259, 1122, 951, 838, $779 \mathrm{~cm}^{-1}$; HRMS (ESI), m/z 318.1933 (calcd for $\left.\mathrm{C}_{16} \mathrm{H}_{29} \mathrm{NO}_{2} \mathrm{Si}+\mathrm{Na}: 318.1860\right)$.

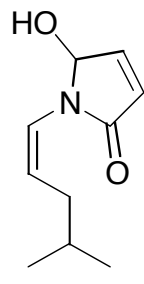

(Z)-5-Hydroxy-1-(4-methyl-1-pentenyl)-1,5-dihydro-2-pyrrolone [( $Z$ )-3]. A solution of (Z)-8 (100 mg, $0.34 \mathrm{mmol})$ in $20 \mathrm{~mL}$ THF was cooled to $-20{ }^{\circ} \mathrm{C}$. A solution of tetrabutylammonium fluoride (1.0 M in THF, $0.44 \mathrm{~mL}, 0.44 \mathrm{mmol}$ ) was added dropwise by syringe over 5 min at this temperature. The reaction mixture was stirred $10 \mathrm{~min}$ at $-20{ }^{\circ} \mathrm{C}$ and was quenched by the addition of ice water. The mixture was extracted with ethyl acetate $(3 \times 15 \mathrm{~mL})$. The combined organic extracts were dried $\left(\mathrm{Na}_{2} \mathrm{SO}_{4}\right)$ and concentrated. The residue was purified by flash chromatography $(1.5 \times 5 \mathrm{~cm}$ silica, 1:1 EtOAc/hexane) to afford pure $(Z)-3(60 \mathrm{mg}, 94 \%)$ as a colorless oil: ${ }^{1} \mathrm{H} \mathrm{NMR}\left(\mathrm{CDCl}_{3}, 500 \mathrm{MHz}\right) \square$ $7.0(\mathrm{dd}, 1 \mathrm{H}, J=6.0,1.6 \mathrm{~Hz}, \mathrm{C} 4-\mathrm{H}), 6.16(\mathrm{dt}, 1 \mathrm{H}, J=9.4,1.6 \mathrm{~Hz}, \mathrm{C} 1 \mathrm{H}), 6.10$ (dd, $1 \mathrm{H}, J=6.0,1.7 \mathrm{~Hz}$, C3-H), 5.70 (br d, 1H, $J=10.2 \mathrm{~Hz}, \mathrm{C} 5-\mathrm{H}), 5.08$ (dt, 1H, $J=9.4,7.4 \mathrm{~Hz}, \mathrm{C} 2 G \mathrm{H}), 3.40$ (br, 1H, OH), $2.09(\mathrm{ABm}, 2 \mathrm{H}, \mathrm{\square}=49.2 \mathrm{~Hz}, \mathrm{C} 3 \mathrm{GH}), 1.67-1.63(\mathrm{~m}, 1 \mathrm{H}, \mathrm{C} 4 \mathrm{G}), 0.91\left(\mathrm{~d}, 3 \mathrm{H}, J=6.7 \mathrm{~Hz}, \mathrm{CH}_{3}\right), 0.89$ $\left(\mathrm{d}, 3 \mathrm{H}, J=6.7 \mathrm{~Hz}, \mathrm{CH}_{3}\right) ;{ }^{13} \mathrm{C} \mathrm{NMR}\left(\mathrm{CDCl}_{3}, 125 \mathrm{MHz}\right) \square 168.4,146.2,127.9,119.6,119.2,83.8,36.2$, 28.7, 22.4, 22.3; IR (neat) $\square_{\max } 3336,2956,2871,1695,1418,1381,1227,1119,1085,979,863,805$ $\mathrm{cm}^{-1}$; HRMS (ESI), $\mathrm{m} / z 204.0992$ (calcd for $\mathrm{C}_{10} \mathrm{H}_{15} \mathrm{NO}_{2}+\mathrm{Na}$ : 204.0995). 


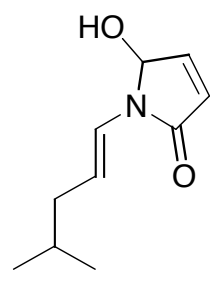

$(E)-5-H y d r o x y-1-(4-m e t h y l-1-p e n t e n y l)-1,5-d i h y d r o-2-p y r r o l o n e ~[(E)-3]$. Following the procedure for the preparation of $(Z)-\mathbf{3}$, a solution of $(E)-8(180 \mathrm{mg}, 0.61 \mathrm{mmol})$ was treated with $n$ $\mathrm{Bu}_{4} \mathrm{NF}(0.74 \mathrm{~mL}, 0.74 \mathrm{mmol})$ to affor $(E)-3(101.2 \mathrm{mg}, 91 \%)$ as a white solid: ${ }^{1} \mathrm{H}$ NMR $\left(\mathrm{CDCl}_{3}, 500\right.$ $\mathrm{MHz}) \square 6.95(\mathrm{dd}, 1 \mathrm{H}, J=6.0,1.5 \mathrm{~Hz}, \mathrm{C} 4-\mathrm{H}), 6.54(\mathrm{~d}, 1 \mathrm{H}, J=14.6, \mathrm{C} 1 \exists \mathrm{H}), 6.04(\mathrm{dd}, 1 \mathrm{H}, J=6.0,0.7$ $\mathrm{Hz}, \mathrm{C} 3-\mathrm{H}), 5.60$ (br s, 1H, C5-H), 5.43 (dt, 1H, $J=14.6,7.4 \mathrm{~Hz}, \mathrm{C} 2 \exists \mathrm{H}), 3.70$ (br, 1H, OH), 1.94 $(\mathrm{ABm}, 2 \mathrm{H}, \mathrm{C} 3 \sqsupset \mathrm{H}), 1.64-1.59(\mathrm{~m}, 1 \mathrm{H}, \mathrm{C} 4 \mathrm{H}), 0.88\left(\mathrm{~d}, 3 \mathrm{H}, J=6.7 \mathrm{~Hz}, \mathrm{CH}_{3}\right), 0.87(\mathrm{~d}, 3 \mathrm{H}, J=6.7 \mathrm{~Hz}$, $\left.\mathrm{CH}_{3}\right) ;{ }^{13} \mathrm{C} \mathrm{NMR}\left(\mathrm{CDCl}_{3}, 125 \mathrm{MHz}\right) \square$ 167.4, 146.1, 127.8, 121.2, 112.6, 83.0, 39.7, 28.8, 22.2; IR (neat) $\square_{\max } 3354,2943,1684,1602,1408,1349,1255,1114,950,867,803,738,703 \mathrm{~cm}^{-1}$; HRMS (ESI), $m / z 204.1035$ (calcd for $\mathrm{C}_{10} \mathrm{H}_{15} \mathrm{NO}_{2}+\mathrm{Na}$ : 204.0995).

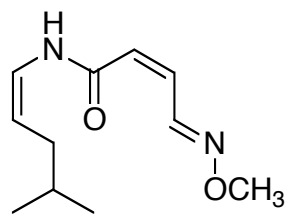

(Z)-4-Methoxyimino-2-butenoic acid ((Z)-4-methyl-1-pentenyl)amide $[(Z)-5]$. A solution of $(Z)-3(15.0 \mathrm{mg}, 0.083 \mathrm{mmol})$, methoxylamine hydrochloride $(62.4 \mathrm{mg}, 0.75 \mathrm{mmol})$ and $\mathrm{Et}_{3} \mathrm{~N}(0.13 \mathrm{~mL}, 0.9 \mathrm{mmol})$ in $\mathrm{MeOH}(5 \mathrm{~mL})$ was warmed at $70{ }^{\circ} \mathrm{C}$ for $3 \mathrm{~h}$. The solvent was removed and the residue was purified by flash chromatography $(1.0 \times 10 \mathrm{~cm}$ silica, $1: 3 \mathrm{EtOAc} /$ hexane $)$ to afford pure (Z)-5 (13.7 mg, 78\%) as a white solid: ${ }^{1} \mathrm{H}$ NMR (acetone- $\left.\mathrm{d}_{6}, 400 \mathrm{MHz}\right) \square 9.09(\mathrm{~d}, 1 \mathrm{H}, J=10.2$, $\mathrm{Hz}, \mathrm{C} 5-\mathrm{H}), 8.92(\mathrm{br}, 1 \mathrm{H}, \mathrm{NH}), 6.81-6.76(\mathrm{~m}, 1 \mathrm{H}, \mathrm{C} 1 \mathrm{H})$ [in the presence of $\mathrm{D}_{2} \mathrm{O}: 6.79(\mathrm{~d}, 1 \mathrm{H}, J=$ 9.1)], $6.47(\mathrm{dd}, 1 \mathrm{H}, J=11.4,10.2 \mathrm{~Hz}, \mathrm{C} 4-\mathrm{H}), 6.27(\mathrm{dd}, 1 \mathrm{H}, J=11.4,5.2 \mathrm{~Hz}, \mathrm{C} 3-\mathrm{H}), 4.79(\mathrm{dt}, 1 \mathrm{H}, J=$ 9.1, $7.9 \mathrm{~Hz}, \mathrm{C} 2 \mathrm{G}), 3.87\left(\mathrm{~s}, 3 \mathrm{H}, \mathrm{CH}_{3} \mathrm{O}\right), 2.02-1.97(\mathrm{~m}, 2 \mathrm{H}, \mathrm{C} 3 \mathrm{G}), 1.66-1.60(\mathrm{~m}, 1 \mathrm{H}, \mathrm{C} 4 \mathrm{H}), 0.89(\mathrm{~d}$, $6 \mathrm{H}, J=6.7 \mathrm{~Hz}, \mathrm{C} 5 \mathrm{H}, \mathrm{C} 6 \boxminus \mathrm{H}) ;{ }^{13} \mathrm{C}$ NMR (acetone-d $\left.6,100 \mathrm{MHz}\right) \square 163.4,148.9,135.3,126.8,122.8$, 112.1, 62.8, 35.8, 29.7, 22.8; IR (neat) $\square_{\max } 3285,2949,1642,1510,1210,1120,1042,970,916,820$, $748 \mathrm{~cm}^{-1}$; HRMS (ESI), $m / z 233.1264$ (calcd for $\mathrm{C}_{11} \mathrm{H}_{18} \mathrm{NO}_{2}+\mathrm{Na}: 233.1260$ ).<smiles>CO/N=C/C=C\C(=O)N/C=C/CC(C)C</smiles>

(E)-4-Methoxyimino-2-butenoic acid ((E)-4-methyl-1-pentenyl)amide [(E)-5]. A solution of $(E)-3(24 \mathrm{mg}, 0.13 \mathrm{mmol})$, methoxylamine hydrochloride $(102 \mathrm{mg}, 1.2 \mathrm{mmol})$ and $\mathrm{Et}_{3} \mathrm{~N}(0.2 \mathrm{~mL}, 1.4 \mathrm{mmol})$ in $\mathrm{MeOH}(5 \mathrm{~mL})$ was warmed at $70^{\circ} \mathrm{C}$ for $5 \mathrm{~h}$. The solvent was removed and the residue was purified by flash chromatography $(1.0 \times 10 \mathrm{~cm}$ silica, $1: 3 \mathrm{EtOAc} / \mathrm{hexane})$ to afford pure $(E)-5$ (19.3 mg, 71\%) as a white solid: ${ }^{1} \mathrm{H}$ NMR (acetone-d $\left.6,500 \mathrm{MHz}\right) \square 9.25$ (br, 1H, NH), 9.12 $(\mathrm{dd}, 1 \mathrm{H}, J=10.3,4.2 \mathrm{~Hz}, \mathrm{C} 5-\mathrm{H}), 6.82-6.77(\mathrm{~m}, 1 \mathrm{H}, \mathrm{C} 1 \mathrm{H})$ [in the presence of $\mathrm{D}_{2} \mathrm{O}: 6.79(\mathrm{~d}, 1 \mathrm{H}, J=$ $14.3 \mathrm{~Hz})$ ], 6.51-6.46 (m, 1H, C4-H) [in the presence of $\left.\mathrm{D}_{2} \mathrm{O}: 6.49(\mathrm{dd}, 1 \mathrm{H}, J=11.5,10.3 \mathrm{~Hz})\right], 6.14$ $(\mathrm{dd}, 1 \mathrm{H}, J=11.5,4.2 \mathrm{~Hz}, \mathrm{C} 3-\mathrm{H}), 5.30(\mathrm{dt}, 1 \mathrm{H}, J=14.3,7.4 \mathrm{~Hz}, \mathrm{C} 2 \mathrm{H}), 3.89\left(\mathrm{~s}, 3 \mathrm{H}, \mathrm{CH}_{3} \mathrm{O}\right), 1.96-1.93$ $(\mathrm{m}, 2 \mathrm{H}, \mathrm{C} 3 \mathrm{H}), 1.62-1.58(\mathrm{~m}, 1 \mathrm{H}, \mathrm{C} 4 \mathrm{H}), 0.89$ (d, 6H, $J=6.6 \mathrm{~Hz}, \mathrm{C} 5 \mathrm{H}, \mathrm{C} 6 \boxminus \mathrm{H})$; ${ }^{13} \mathrm{C}$ NMR (acetone$\left.\mathrm{d}_{6}, 125 \mathrm{MHz}\right) \square 162.4,148.4,134.6,126.5,124.5,112.9,62.3,39.8,30.0,22.3$; IR (neat) $\square \max 3260$, 2919, 1631,1531,1461, 1302, 1261, 1114, 1044, 950, 907, 819, $737 \mathrm{~cm}^{-1}$; HRMS (ESI), m/z 233.1258 (calcd for $\mathrm{C}_{11} \mathrm{H}_{18} \mathrm{NO}_{2}+\mathrm{Na}$ : 233.1260). 


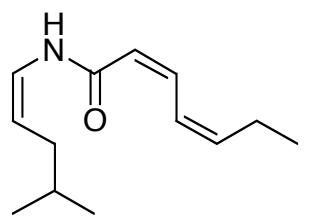

(2Z,4Z)-2,4-Heptadienoic acid ((Z)-4-methyl-1-pentenyl)amide [(Z)-6]. Propyltriphenylphosphonium bromide $(116 \mathrm{mg}, 0.295 \mathrm{mmol})$ was slurried in THF $(10 \mathrm{~mL})$ at $-78^{\circ} \mathrm{C}$ and a solution of $\mathrm{KN}\left(\mathrm{SiMe}_{3}\right)_{2}(0.5 \mathrm{M}$ in toluene, $0.67 \mathrm{~mL}, 0.34 \mathrm{mmol})$ was added dropwise by syringe over 1 min. The resulting orange slurry was warmed to $-20^{\circ} \mathrm{C}$ over $20 \mathrm{~min}$, then cooled to $-78^{\circ} \mathrm{C}$. A solution of $(Z)-3(25 \mathrm{mg}, 0.13 \mathrm{mmol})$ in THF $(5 \mathrm{~mL})$ was added dropwise by syringe over $5 \mathrm{~min}$. The reaction mixture was stirred $30 \mathrm{~min}$ at $-78^{\circ} \mathrm{C}$ and then warmed to $0{ }^{\circ} \mathrm{C}$. The reaction mixture was quenched by the addition of saturated aqueous $\mathrm{NH}_{4} \mathrm{Cl}$, and the mixture was extracted with ether $(3 \times 10 \mathrm{~mL})$. The combined organic extracts were dried $\left(\mathrm{Na}_{2} \mathrm{SO}_{4}\right)$ and concentrated. The residue was purified by flash chromatography $(1.5 \times 5 \mathrm{~cm}$ silica, 1:5 EtOAc/hexane) to afford pure $(Z)-6(26.1 \mathrm{mg}, 94 \%)$ as a colorless oil: ${ }^{1} \mathrm{H}$ NMR (acetone- $\left.\mathrm{d}_{6}, 400 \mathrm{MHz}\right) \square 8.70(\mathrm{br}, 1 \mathrm{H}, \mathrm{NH}), 7.52$ (dd, $1 \mathrm{H}, J=10.8,11.5 \mathrm{~Hz}$, $\mathrm{C} 5-\mathrm{H}), 6.89-6.79(\mathrm{~m}, 2 \mathrm{H}, \mathrm{C} 4-\mathrm{H}, \mathrm{C} 1 \mathrm{GH})$ [in the presence of $\mathrm{D}_{2} \mathrm{O}: 6.87(\mathrm{dd}, 1 \mathrm{H}, J=11.4,11.5 \mathrm{~Hz}, \mathrm{C} 4-$ $\mathrm{H}), 6.82(\mathrm{~d}, 1 \mathrm{H}, J=8.7 \mathrm{~Hz}, \mathrm{C} 1 \exists \mathrm{H})], 5.90(\mathrm{~d}, 1 \mathrm{H}, J=11.4, \mathrm{C} 3-\mathrm{H}), 5.80(\mathrm{dt}, 1 \mathrm{H}, J=10.8,8.6 \mathrm{~Hz}, \mathrm{C} 6-$ $\mathrm{H}), 4.72(\mathrm{dd}, 1 \mathrm{H}, J=8.7,7.8 \mathrm{~Hz}, \mathrm{C} 2 \exists \mathrm{H}), 2.30-2.24$ (m, 2H, C7-H), 2.02-1.98 (m, 2H, C3GH), 1.66$1.62(\mathrm{~m}, 1 \mathrm{H}, \mathrm{C} 4 \mathrm{H}), 1.01(\mathrm{t}, 3 \mathrm{H}, J=7.5 \mathrm{~Hz}, \mathrm{C} 8-\mathrm{H}), 0.91(\mathrm{~d}, 6 \mathrm{H}, J=6.6 \mathrm{~Hz}, \mathrm{C} 5 \boxminus \mathrm{H}, \mathrm{C} 6 \boxminus \mathrm{H}) ;{ }^{13} \mathrm{C} \mathrm{NMR}$ (acetone- $\left.\mathrm{d}_{6}, 100 \mathrm{MHz}\right) \square 164.4,141.9,137.2,125.9,123.1,121.3,110.6,35.8,29.8,22.9,21.4,14.7$; IR (neat) $\square_{\max } 3309,2957,1639,1456,1411,1346,1232,1146,1016,867,826,739 \mathrm{~cm}^{-1}$; HRMS (ESI), $m / z 230.1516$ (calcd for $\mathrm{C}_{13} \mathrm{H}_{21} \mathrm{NO}+\mathrm{Na}$ : 230.1515).

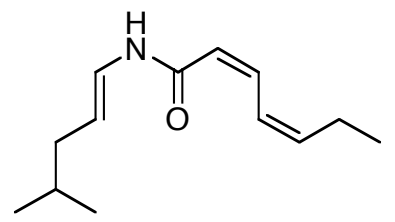

$(2 Z, 4 Z)-2,4-H e p t a d i e n o i c ~ a c i d ~((E)-4-m e t h y l-1-p e n t e n y l)$ amide $[(E)$ 6]. Following the procedure for the preparation of $(Z)-6$, propyltriphenylphosphonium bromide (116 $\mathrm{mg}, 0.295 \mathrm{mmol}), \mathrm{KN}\left(\mathrm{SiMe}_{3}\right)_{2}(0.5 \mathrm{M}$ in toluene, $0.67 \mathrm{~mL}, 0.34 \mathrm{mmol})$ and 11 (25 $\left.\mathrm{mg}, 0.13 \mathrm{mmol}\right)$ afforded pure $(E)-6(24.9 \mathrm{mg}, 90 \%)$ as a white solid: ${ }^{1} \mathrm{H}$ NMR (acetone-d $\left.6,500 \mathrm{MHz}\right) \square 8.98(\mathrm{br}, 1 \mathrm{H}$, $\mathrm{NH}), 7.51(\mathrm{dd}, 1 \mathrm{H}, J=11.1,11.3 \mathrm{~Hz}, \mathrm{C} 5-\mathrm{H}), 6.87-6.77(\mathrm{~m}, 2 \mathrm{H}, \mathrm{C} 4-\mathrm{H}, \mathrm{C} 1 \mathrm{G} \mathrm{H})$ [in the presence of $\mathrm{D}_{2} \mathrm{O}$ : $6.86(\mathrm{dd}, 1 \mathrm{H}, J=11.4,11.3 \mathrm{~Hz}, \mathrm{C} 4-\mathrm{H}), 6.81(\mathrm{~d}, 1 \mathrm{H}, J=14.3 \mathrm{~Hz}, \mathrm{C} 1 \boxminus \mathrm{H})], 5.79(\mathrm{dt}, 1 \mathrm{H}, J=11.1,8.4$ Hz, C6-H), 5.75 (d, 1H, J=11.4 Hz, C3-H), 5.22 (dt, 1H, J=14.3, 7.4 Hz, C2 $\mathrm{H}), 2.31-2.27$ (m, 2H, C7-H), 1.94-1.91 (m, 2H, C3GH), 1.63-1.55 (m, 1H, C4GH), $1.01(\mathrm{t}, 3 \mathrm{H}, J=7.5 \mathrm{~Hz}, \mathrm{C} 8-\mathrm{H}), 0.89$ (d, $6 \mathrm{H}, J=6.6 \mathrm{~Hz}, \mathrm{C} 5 \boxminus \mathrm{H}, \mathrm{C} 6 \boxminus \mathrm{H}) ;{ }^{13} \mathrm{C}$ NMR (acetone-d $\left.6,125 \mathrm{MHz}\right) \square 163.5,141.3,136.6,125.4,124.9$, 121.0, 111.4, 40.0, 30.1, 22.4, 21.0, 14.3; IR (neat) $\square_{\max } 3272,2955,1643,1525,1461,1402,1302$, 1243, 1161, $950 \mathrm{~cm}^{-1}$; HRMS (ESI), $m / z 230.1512$ (calcd for $\mathrm{C}_{13} \mathrm{H}_{21} \mathrm{NO}+\mathrm{Na}: 230.1515$ ). 